\title{
Transcatheter closure for post-infarction ventricular septal defect: A meta-analysis of the current evidence
}

\author{
Xiang Yang ${ }^{1}$, Zeran $\mathrm{Yu}^{2}$, Yu Wang ${ }^{3}$, Yunchuan Ding ${ }^{1}$, Ruizhi $\mathrm{Ni}^{3}$, and Pingxi Xiao ${ }^{4}$ \\ ${ }^{1}$ Yanan Hospital of Kunming City, Yunnan Cardiovascular Hospital \\ ${ }^{2}$ The Second People's Hospital of Yunnan Department \\ ${ }^{3} 1$ st Hospital of Kunming Medical University \\ ${ }^{4}$ Sir Run Run Hospital of Nanjing Medical University
}

November 5, 2020

\begin{abstract}
Objective: Post-infarction ventricular septal defect (PIVSD) is a severe complication of acute myocardial infarction. Transcatheter closure (TCC) perform an alternative option to the surgical repair. This study was undertaken to examine the published literature to give the objective evidence of TCC using a meta-analysis. Methods: We searched for significant medical and publishers' databases. Two reviewers checked the quality of studies and extracted data. Eligible studies included single-arm studies and comparative studies. Weighted mean, pooled event rates, efficacy outcomes and odds ratios(OR) for immediate shunt reduction(ISR), presence of cardiogenic shock (CS), New York Heart Association ( NYHA) class IV, time from AMI to ventricular septal defect(VSD), time to VSD closure was estimated. Results: 27 single arm articles (462 patients) were included. The pooled event rate was $89.7 \%$ (95\%CI: 0.772-1.021) for successful device implantation, $80.9 \%$ (95\%CI: 0.645-0.972) for ISR, $31.5 \%$ (95\% CI of $0.149-0.482$ ) for 30-day mortality, $25.3 \%$ (95\%CI: 0.072-0.434) for 30-day mortality of primary closure at acute phase. CS (OR=3.607, 95\%CI: 2.301-5.653), NYHA class IV (OR=6.491, 95\%CI: 1.444-29.188) and time to VSD closure are risk predictor for TCC. There is no correlation between the defect size $(\mathrm{OR}=2.592,95 \% \mathrm{CI}$ : 0.380-17.661) and mortality. Conclusion: TCC should be a relatively safe and low invasive method for PIVSD, with an excellent successful device implantation rate and acceptable low 30-day mortality. The procedure appears promising, but its safety and efficacy could only be demonstrated by randomized controlled trials. Therefore, they are needed more investigations to determine whether the acute phase or chronic phase to practice the procedure.
\end{abstract}

\section{Introduction}

Post-infarction ventricular septal defect (PIVSD) is a rare and devastating complication of acute myocardial infarction (AMI), the prevalence rate is $i 1 \%^{[1]}$. Even though the patients get prompt medical management, the mortality rate is higher than $90 \%{ }^{[1-2]}$. Until recently, surgery repair as a comprehensive applicant and a generally safe procedure is associated with high morbidity and mortality $(20-81 \%)^{[1,3]}$. On the contrary, medical therapy alone has a minimal survival rate of less than $8 \%$ at 30 days ${ }^{[4]}$ and less than $3 \%$ at one year ${ }^{[1,2,4,5]}$. Even though the surgical closure presents a modest improvement of mortality compared with medical therapy, surgical intervention own self-limitations like restriction of advanced age, multi perioperative morbidity and surgical expertise are not readily available. It is suggested that the transcatheter closure (TCC) of PIVSD as promising alternative management to cause the less invasive and be recommended by authoritative national guidelines ${ }^{[6-8]}$. Evolution of the timing of TCC can be broadly categorized into 2 phases: The acute phase occurs within the first two weeks after AMI, which is characterized by coagulation necrosis and release of lytic enzymes from neutrophils resulting in myocardial necrosis; The chronic phase begins after $3-4$ weeks and is characterized by fibrosis and scar formation around the edges of the defect. To our knowledge, the actual benefits of TCC proposed for use in the PIVSD remain uncertain. Moreover, the 
majority of clinical proof is based on limited study sizes. We, therefore, systemically searched and analyzed the available literature to provide physicians and patients an objective estimate of the outcomes of TCC on PIVSD patients in the current era.

\section{Methods}

\subsection{Data sources}

To identify eligible studies, we searched the electronic databases from 1988 until July 2019 through two investigators. The electronic database includes the PubMed, EMBASE, MEDLINE, Ovid's MEDLINE, CENTRAL and ClinicalTrials.gov, Web of Science, Cochrane databases and EBM review, using various combinations of Medical Subject Headings (MeSH) and text words including: "ventricular septal defect(VSD)", "ventricular septal rupture", "myocardial infarction", "closure", "septal occlude device", "transcatheter", "percutaneous".

\subsection{Selection criteria}

We did not apply any regions, publication types, language restrictions. Articles were included if they: i) reported the 30-day or in-hospital mortality; ii) Case reports and case series had to include limited people ([?]4) in each study. According to the selection criteria, 27 articles meet the strict criteria. A PRISMA flow chart depicts the process of search and selection of studies shown in Figure 1.

\subsection{Outcomes of interest}

For the single-arm study, the primary outcome was the pooled event rates for successful device implantation rate, 30-day mortality rate and 30- day mortality for primary closure at the acute phase, and secondary outcomes were the pooled event rate of immediate shunt reduction(ISR). While, odds ratios (OR) for ISR, presence of cardiogenic shock(CS), n class IV, time from AMI to VSD, time to VSD closure were estimated.

For the pairwise comparison with surgical closure, the primary outcome was 30-day mortality rate and secondary outcomes were immediate shunt reduction.

\subsection{Study enrollment and data extraction}

Two reviewers (Xiang Yang, Ze ran Yu) extracted data independently using a predefined data extraction form. Disagreements were resolved by discussion or consensus with a third reviewer. Microsoft Office Excel 2013 (Microsoft, Redmond, Washington) was used for data extraction. The data extracted included the study name; publication year, study design, country of publication, sample size and baseline characteristics (age, gender, prior surgical history, time from AMI to VSD, time to VSD closure, when to intervention, presence of CS, prior intra-aortic balloon pump (IABP), inotropes use and size of the defect), and procedural characteristics (successful device implantation, size of device, type of device, immediate shunt reduction), the adverse events and complications. For studies with insufficient information, the reviewers contacted the primary authors, when possible, to acquire and verify the data.

\subsection{Data analyses}

Dichotomous data were analyzed by using the pooled event rates and

OR computed using the Mantel Haenszel method (with 95\% Confidence Interval(CI)). Continuous outcomes measured on the same scale were expressed as a mean value and standard deviation and were analyzed by using weighted mean. I-square $\left(\mathrm{I}^{2}\right)$ test was performed to assess the impact of study heterogeneity on the results of the meta-analysis. According to the Cochrane review guidelines, if severe heterogeneity was present at $\mathrm{I}^{2}>50 \%$, the random effect models were chosen. Otherwise, the fixed effect models were used. $\mathrm{Z}$ value $(\mathrm{Z})$ is the overall effect. Moreover, a sensitivity analysis was conducted by deleting each study individually to evaluate the quality and consistency of the results. Visual inspection of the Begg's test was done to assess publication bias. Stata 14.0 (Stata Corp LLC, Texas, USA) was used for statistical analysis.

\section{Results 3.1. Selected studies}


Among 661 retrieved searched articles, 27 articles were finally eligible with a total of 462 included patients, 23 single arm studies and 4 comparative studies were identified which included a total of 329 and 133 patients respectively. These articles were published from $1998^{[9]}$ to $2018^{[10-11]}$. The mean age was $68.5 \pm 2.3$ years ( $\mathrm{n}=403$; median:68.0), 52.4\% are males(196/373).

\subsection{Study quality}

The results of the Newcastle-Ottawa scale assessment are 13 studies,eight stars, four studies seven stars, nine studies six stars, and one studies five stars(shown in Supplementary Tab. 1) ${ }^{[12]}$.

\subsection{Quantitative meta-analyses findings}

\subsubsection{Basic characteristics}

Among 462 patients with PIVSD, 47.2\% had CS (95\%CI: 0.267-0.676) patients, which results from 18 included articles (155/333). About 35.8\% (95\%CI: 0.114- 0.602) patients needed IABP and 49.4\%(95\%CI: 0.217 - 0.772) needed inotropes,seventeen $(192 / 325)$ and twelve $(102 / 195)$ included studies were enrolled. Coronary single-vessel, double-vessel and triple-vessel disease were present in $48.9 \%(132 / 270), 34.8 \%$ $(94 / 270)$, and $16.3 \%(44 / 270)$ of patients, respectively. Revascularization and Prior surgical closure rate was $39.4 \%$ (95\%CI: $0.221-0.568)$ and $80.9 \%$ (95\%CI: $0.010-0.355)$, respectively eighteen $(119 / 331)$ and twenty-one(71/333) included articles were assessed for this outcome. The rate of primary closure at the acute phase reported in twenty-one studied (155/360) was $29.7 \%$ (95\%CI: $0.095-0.499)$. The weighted mean results were as follows: time from AMI to VSD and time to VSD closure was 2.6 days, 17.3 days. The defect size and device size were $13.1 \mathrm{~mm}$ and $19.6 \mathrm{~mm}$. The clinical follow-up time was 589.8 days. Table 1 summarizes the initial assessment results of 27 studies.

\subsubsection{Occluder devices}

A considerable amount of literature has been discussed and analyzed the device for TCC of a PIVSD. The most commonly used occlude devices are as follows: atrial-septal-defect occluder (ASDo, 118/355, 33.2\%), muscular ventricular-septal-occluder (mVSDo, 96/355, 27.0\%) and a specific post-infarction mVSDo occluder (PimVSDo, 131/355, 36.9\%) which is specifically designed for the PIVSD. There was minority studies used device(10/355, 2.8\%): patent ductus arteriosus occluder ${ }^{[10]}$ (PDAo,3/10,0.3\%), Amplatzer cribriform occluder $^{[13]}(\mathrm{ACO}, 2 / 10,0.2 \%)$, modified double-disc occluder ${ }^{[14]}(3 / 10.0 .3 \%)$ and other device ${ }^{[15]}(2 / 10,0.2 \%)$.

\subsubsection{Efficacy Outcomes}

The results of the pooled event rate come from 27 articles. 30- day mortality was 31.5\% (95\%CI of 0.149- 0 . 482, (shown in Fig. 2A)), all patients were assessed for this outcome. 24 out of 27 articles(370/415), overall 415 patients were treated medically, and the successful device implantation rate was $89.7 \%$ (95\% CI: 0.772 1.021, (shown in Fig. 2B)). From 21 studies (274/370), we got the pooled event rate of immediate shunt reduction was $80.9 \%$ (95\%CI: 0.645- 0.972, (shown in Fig. 2C)). Eighteen included articles (75/270) were assessed for the pooled event rate of 30-day mortality of primary closure at the acute phase, which was $25.3 \%$ (95\%CI: 0.072-0.434, (shown in Fig. 2D)). Table 2 summarizes the original assessment results of single-arm studies.

On pairwise meta-analysis, no difference in 30-day mortality was identified ( $\mathrm{OR}=0.920$ (95\%CI: 0.420-2.013; $\mathrm{p}=0.252$; (shown in Supplementary Fig. A)), no difference in immediate shunt reduction rate was identified $(\mathrm{OR}=6.213$ (95\%CI: 2.085-18.511; $\mathrm{p}=0.092$; (shown in Supplementary Fig. B)).

\subsubsection{Predictors of mortality after transcatheter PIVSD closure}

Individual analyses of predictors of mortality after transcatheter PIVSD closure based on the random effect showed good results. From four included articles (Zhang et al ${ }^{[16]}$, Egbe et al ${ }^{[17]}$, Assenza et al ${ }^{[18]}$, Calvert et $\left.\mathrm{al}^{[15]}\right)$, CS (OR=3.607, 95\%CI: 2.301-5.653; $\mathrm{p}=0.000$, (shown in Fig. 3A))incline to add the risk of mortality, Zhang et al. and Calvert et al. studies show New York Heart Association (NYHA) class IV (OR=6.491, 95CI: 1.444-29.188; $\mathrm{p}=0.015$, shown in Fig. 3B) was a predictor for TCC, time to PIVSD closure (OR=0.850, 
95CI: $0.737-0.980 ; \mathrm{p}=0.025$, shown in Fig. $3 \mathrm{C}$ ) was also the risk factors for PIVSD closure patients. There is no direct correlation between the defect size ( $\mathrm{OR}=2.592$, 95\%CI: $0.380-17.661$, shown in Fig. 3D) and death.

\subsubsection{Complications}

23 included articles $(\mathrm{n}=415)$ referred to the major immediate procedure-related complications, which included: device dislocation $(\mathrm{n}=10,2.41 \%)$, device embolization $(\mathrm{n}=7,1.69 \%)$, left ventricular rupture or perforation $(\mathrm{n}=12,2.89 \%)$, hemolysis $(\mathrm{n}=12,2.89 \%)$, blood transfusion $(\mathrm{n}=15,3.61 \%)$, arrhythmia $(\mathrm{n}=20,4.82 \%)$, residual $\operatorname{shunt}(\mathrm{n}=11,2.65 \%)$. The complications not related to the procedure are as follows: multi-organ failure $(\mathrm{n}=19,4.58 \%)$, respiratory(renal) and/or circulatory failure $(\mathrm{n}=20,4.82 \%)$, cerebral infarction( $\mathrm{n}=3$, $0.72 \%)$.

\subsection{Publication bias}

The Begg's test ${ }^{[19-20]}$ of the 30-day mortality and successful device implantation rate indicated there might be mild publication bias (shown in Fig. 4A and 4B), which may also be caused by a small sample size or single-arm studies.

\section{Discussion}

4.1 Closure and visual improvement rates according to treatment

Transcatheter closure of VSD was first described by Lock $^{[21]}$ in 1988. In the last four decades, TCC for PIVSD has justified a feasible and less invasive procedure. Our results show that the successful device implantation rate was $89.7 \%$ (95\%CI: $0.772-1.021)$. The 30 - day mortality rate was approximately $31.5 \%$ (95\%CI of 0.149- 0. 482) and as high as $25.3 \%$ (95\%CI: $0.072-0.434)$ when primary closure at the acute phase ( $\mathrm{i} 2$ weeks). It is comparatively lower than the surgical repair treatment within three weeks and more prolonged than three weeks ,respectively $50 \%$ and $38 \%{ }^{[22-23]}$. Data from several sources have identified the patients who underwent successful percutaneous closure showed a trend toward a satisfactory long-term outcome $e^{[24-25]}$.

In the comparison of TCC to surgical closure, the pooled analysis of two studies showed no significant difference in 30-day mortality, immediate shunt reduction. The small number of studies limits these conclusions. It is of necessity for future randomized controlled trials to demonstrate TCC compared with surgical closure.

\subsection{Spontaneous PIVSD closure}

What we know about the option of occluder device is primarily based upon empirical studies that the conventional devices were ASDo, mVSDo and PimVSDo. There were no related articles about the comparison of varieties of devices, so it is uncertain which type of device is the best choice for catheterization. However, surveys such as those conducted by Szkutnik et al ${ }^{[24]}$ and Wacinski et al ${ }^{[25]}$ have shown that PimVSD is more suitable for PIVSD owing to the particular structure (wider waist, larger disks and a denser construction ) could occlude moderate to severe shunt in a short period time.

The location and size of the device were detected by two-dimensional transthoracic echocardiography (2DTTE) in a large number of studies, with a sizing balloon to determine the size in minor cases. However, it is taking the risk of enlargement of the defect, whether this method can be adopted depends on the firmness of the rims and the anatomic morphology of $\mathrm{VSD}^{[15,26]}$. Recently, several attempts have been made to use three-dimensional transthoracic echocardiography (3DTTE) or three-dimensional transesophageal echocardiography (3DTEE) to assess the VSD. Many analysts now argue that the strategy of 3DTTE/3DTEE over 2DTTE has been successful for the evaluation of VSD following AMI, for example, argues that VSD shape and its changes during the cardiac cycle can be visually and quantitatively displayed. 3DTTE/3DTEE may thus be particularly useful before and during percutaneous VSD closure ${ }^{[27-29]}$.

There is still no definite conclusion about how to select the size of the device. Data from several experienced articles have identified the optimal diameter should be twice the size of the measured defect diameter or 
at least $10 \mathrm{~mm}$ larger,it can prevent incomplete closure or dislocation and embolization of the device due to continued septal necrosis in the acute phase ${ }^{[30-31]}$, in turn, the occluder size was slightly larger than the measurable defect size in the chronic phase ${ }^{[2]}$. As for the best timing of TCC after AMI, there were two contrary opinions, majorities of people hold the idea that the delay of 10-14 days may help for shaping the scarred tissue to restore the perforation tissue and improving the NYHA classification ,so that can acquire the better progression ${ }^{[16]}$. The others consider the percutaneous closure should do as quickly as possible because of prolonging the time was not associated with the low mortality ${ }^{[15]}$. However, there was no direct evidence to justify which choice could decrease the mortality effective. When treated with PIVSD, one-key to success in procedure was immediate complete or partial shunt reduction.Our data confirmed about $80.9 \%$ shunt got to reduce after interventional surgery. Just like Calvert et al ${ }^{[15]}$ indicated that if the shunt cannot decrease at least two thirds, the patients are most unlikely to survive either to surgery or to discharge. Moreover, there is no apparent distinction between a variety of occlusion devices, which depends on the edge condition and size of defects identified by echocardiography. As a rigorous surgical procedure requiring expertise and collaboration between interventionists, anesthetists, and echocardiography specialists.

\subsection{Factors affecting TCC outcomes for PIVSD}

Cardiogenic shock(CS) is a terrible complication of PIVSD accompanied by the high mortality rate is approximately $89 \%^{[2]}$. Our data suggest that patients who developed VSD and died were the more likely to the occurrence of CS before TCC. Accompanied by catastrophic mortality (about 87\%) and reduced progression on patients with CS also in the SHOCK trial ${ }^{[1]}$. Similarly, majorities studies have confirmed the same conclusion ${ }^{[10,15,18,32]}$. In high surgical-risk patients with PIVSD, NYHA class IV and a long time from VSD to transcatheter are also correlated with high mortality, while the defect size and have no relevance to the mortality. There may be a variety of mechanisms for these results.

On the one hand, When NYHA class IV indicates the patients with a terrible clinical state, they need the support of inotropes or IABP, even the surgical repair promptly. Now keeping the stability of the hemodynamics is a critical therapeutic strategy at an early stage of PIVSD. On the other hand, and it is hard to find available occlude devices and lack of firm tissue to seat the device for large defect sizes. Unpaired occluder will generate resident shunt and long-term residual shunt can lead to infective endocarditis and even left ventricular rupture ${ }^{[33,34]}$. Moreover, in their studies, Shi Tai et al. Calvert et al. found that advanced age and female sex are risk factors for PIVSD patients. The following explanation about why women with increased risk of VSD is given by Andrew: "Contributing factors may include women's older age at presentation, greater comorbidities, frequency of atypical symptoms leading to treatment delay, and decreased likelihood of treatment with guideline-driven medical and reperfusion therapies ${ }^{[35,36]}$ ".

\subsection{Adverse events and complications}

The major immediate procedure-related adverse events and complications, which related to include the closure procedure were: device dislocation, device embolization, left ventricular rupture or perforation, hemolysis, blood transfusion, arrhythmia, residual shunt. The complications not related to the procedure are as follows: multi-organ failure, respiratory(renal) and/or circulatory failure, cerebral infarction. In the final part of the Theses, Risseeuw writes: " Procedure-related complications did not occur frequently and were rarely the direct cause of mortality, but mortality occurring in the long-term period following intervention was often the result of one of these complications ${ }^{[37]}$." Residual shunt as one of the relatively common complications was caused by progressive septal necrosis or other complications of relevance to device placement. It was qualitatively categorizing into trivial, mild, moderate and severe. Nevertheless, it is difficult to obtain a uniform standard due to empirical judgment with the naked eye. Recent evidence demonstrated that three-dimensional color Doppler echocardiography is a feasible method for quantifying the shunt volume in VSD patients ${ }^{[27]}$, which had potential value on judging the indication of residual shunt need a secondary percutaneous procedure or surgical intervention. In order to reduce the occurrence of adverse events and complications, there is no doubt that the physicians should take care and comprehensive into consideration the medical conditions of patients to assess which is the best therapeutic decision. 


\subsection{Study limitations}

Due to the lack of the control group and are non-randomized data, this paper cannot provide accurate and comprehensive meta-analysis results. Based on the rare case of post-infarction VSD and the difference of every case, it is beyond the possibility of this series of studies to practice under the randomized controlled trials (transcatheter closure v.s. surgical closure). The reader should bear in mind that the study is based on smaller sample sizes of PIVSD patients. Furthermore, there is an evitable selection bias among these cases.

5. Conclusion

In conclusion, transcatheter closure is a feasible and time-saving procedure for selected post-infarction VSD patients. Accompanying with a satisfactory device implantation rate and less invasive makes good results. Different degrees of reduced immediate resident shunts kept the stability of the hemodynamics and decreased mortality. However, the mortality rate remains high in cardiogenic patients. Further studies referring to more patients are needed on the choice of surgical approach and assessing the timing of intervention is necessary. Furthermore, we would like to update this meta-analysis if the number of articles in this field is much more abundant in the future.

\section{References}

[1]Crenshaw BS, Granger CB, Birnbaum Y, et al. Risk factors, angiographic patterns, and outcomes in patients with ventricular septal defect complicating acute myocardial infarction.GUSTO-I (Global Utilization of Streptokinase and TPA for Occluded Coronary Arteries) Trial Investigators. Circulation.2000;101:27-32.

[2] Menon V, Webb JG, Hillis LD, et al. Outcome and profile of ventricular septal rupture with cardiogenic shock after myocardial infarction: a report from the SHOCK Trial Registry. Should we emergently revascularize Occluded Coronaries in cardiogenic shock? J Am Coll Cardiol. 2000;36(3 Suppl A):1110-1116.

[3]Pretre R, Ye Q, Grunenfelder J, Lachat M, Vogt PR, Turina MI. Operative results of "repair" of ventricular septal rupture after acute myocardial infarction. Am J Cardiol 1999;84:785-788.

[4] Omar S, Morgan GL, Panchal HB, et al. Management of post-myocardial infarction ventricular septal defects: a critical assessment. J Interv Cardiol. 2018;31(6):939-948.

[5]Labrousse L, Choukroun E, Chevalier JM, et al. Surgery for post infarction ventricular septal defect:risk factors for hospital death and long term results. Eur J Cardiothorac Surg. 2002;21:725-731.

[6]Antman EM, Anbe DT, Armstrong PW, et al. ACC/AHA guidelines for the management of patients with ST-elevation myocardial infarction-Executive summary. A report of the American College of Cardiology/American Heart Association Task Force on Practice Guidelines (Writing Committee to revise the 1999 guidelines for the management of patients with acute myocardial infarction). J Am Coll Cardiol 2004;44:671719 .

[7]Van de Werf F, Ardissino D, Betriu A, et al.Management of acute myocardial infarction in patients presenting with ST-segment elevation. The Task Force on the Management of Acute Myocardial Infarction of the European Society of Cardiology. Eur Heart J 2003;24:28-66.

[8]Ibanez B, James S, Agewall S, et al. 2017 ESC Guidelines for the management of acute myocardial infarction in patients presenting with ST-segment elevation. The Task Force for the management of acute myocardial infarction in patients presenting with ST-segment elevation of the European Society of Cardiology (ESC). European Heart Journal. 2017;(00):1-66

[9]Landzberg MJ, Lock JE. Transcatheter management of ventricular septal rupture after myocardial infarction. Semin Thoracic Cardiovasc Surg. 1998;10:128-132

[10]Tai S, Tang JJ, Tang L, et al. Management and Outcome of Ventricular Septal Rupture Complicating Acute Myocardial Infarction: What Is New in the Era of Percutaneous Intervention? Cardiology 
2018;141:226-232. [11]Aggarwal M, Natarajan K, Vijayakumar M, et al. Primary transcatheter closure of post-myocardial infarction ventricular septal rupture using amplatzer atrial septal occlusion device: A study from tertiary care in South India. Indian Heart Journal, 2018, 70(4): 519-527.

[12]Stang A. Critical evaluation of the Newcastle-Ottawa scale for the assessment of the quality of nonrandomized studies in meta analyses. Eur J Epidemiol 2010:25(9):603-605.

[13] Trivedi KR, Aldebert P, Riberi A, et al. Sequential management of post-myocardial infarction ventricular septal defects. Archives of Cardiovascular Diseases, 108(5), 321-330.

[14]Xian-Yang Z, Yong-Wen Q, Ya-Ling Han, et al. Long-term efficacy of transcatheter closure of ventricular septal defect in combination with percutaneous coronary intervention in patients with ventricular septal defect complicating acute myocardial infarction: a multicentre study. EuroIntervention 2013;8:1270-1276.

[15]Calvert PA, Cockburn J, Wynne D, et al. Percutaneous closure of postinfarction ventricular septal defect: in-hospital outcomes and long-term follow-up of UK experience. Circulation. 2014;129(23):23952402.[16]Zhang R, Sun Y, Sun M, et al. In-hospital outcomes and long-term follow-up after percutaneous transcatheter closure of postinfarction ventricular septal defects. Biomed Res Int. 2017;2017:797-1027.

[17]EgbeA C, Poterucha J T, Rihal C S, et al. Transcatheter closure of postmyocardial infarction, iatrogenic, and postoperative ventricular septal defects: The Mayo Clinic experience. Cathet. Cardiovasc. Intervent.2015, 86: 1264-1270.

[18] Assenza GE, McElhinney DB, Valente AM, Pearson DD, Volpe M, Martucci G, et al. Transcatheter closure of post-myocardial infarction ventricular septal rupture. Circ Cardiovasc Interv. 2013 Feb; 6(1): $59-67$.

[19]Egger M,Davey Smith G,Schneider M,et al.Bias in meta analysis detected by a simple,graphical test.BMJ,1997,315:629-634.

[20]Begg CB, Mazumdar M. Operating characteristics of a rank correlation test for publication bias.Biometrics,1994,50:1088-1101.

[21]Lock JE, Block PC, McKay RG, Baim DS, Keane JF.Transcatheter closure of ventricular septal defects. Circulation.1988;78:361-8.

[22]Demkow M, Ruzyllo W, Kepka C, Chmielak Z, Konka M, Dzielinska Z, Wilczynski J, Juraszynski Z. Primary transcatheter closure of postinfarction ventricular septal defects with the Amplatzer septal occluderimmediate results and up-to 5 years follow-up. EuroIntervention. 2005;1:43-47.

[23]Heiberg J, Hjortdal VE, Nielsen-Kudsk JE.. Long-Term Outcome after Transcatheter Closure of Postinfarction Ventricular Septal Rupture. Journal of Interventional Cardiology. 2014; 27(5), 509-515.

[24]Szkutnik M, Bialkowski J, Kusa J, et al. Postinfarction ventricular septal defect closure with Amplatzer occluders. Eur J Cardiothorac Surg. 2003;23(3):323-7.

[25]Wacinski P, Bilodeau L, Ibrahim R. Successful early percutaneous closure of acute ventricular septal rupture complicating acute myocardial infarction with Amplatzer ventricular septal occluder. Cardiology. 2007;14(4):411-4.

[26]Bialkowski J, Szkutnik M, Kusa J, et al. Transcatheter closure of postinfarction ventricular septal defects using Amplatzer devices. Rev Esp Cardiol. 2007;60:548-51.

[27]Tracy E, Zhu M, Streiff C, et al. Quantification of the area and shunt volume of multiple, circular, and noncircular ventricular septal defects: A 2D/3D echocardiography comparison and real time 3D color Doppler feasibility determination study. Echocardiography. 2018;35:90-99.

[28] Hadeed K, Hascoet S, Amadieu R, et al. Assessment of Ventricular Septal Defect Size and Morphology by Three-Dimensional Transthoracic Echocardiography. Journal of the American Society of Echocardiography, 
2016; 29(8), 777-785.

[29] Arisha MJ, Hsiung MC, Nanda NC, et al. Incremental value of live/real time three-dimensional transesophageal echocardiography in the assessment of ventricular septal rupture following acute myocardial infarction. Echocardiography. 2017;34:1680-1686.

[30] Martinez MW, Mookadam F, Sun Y, et al. Transcatheter closure of ischemic and post-traumatic ventricular septal ruptures. Catheter Cardiovasc Interv. 2007;69(3):403-407.

[31]Maltais S, Ibrahim R, Basmadjian AJ, et al. Postinfarction ventricular septal defects: towards a new treatment algorithm? Ann Thorac Surg. 2009;87(3):687-92.

[32]Sulzgruber P, El-Hamid F, Koller L, Forster S, Goliasch G, Wojta J, et al. Long-term outcome and risk prediction in patients suffering acute myocardial infarction complicated by postinfarction cardiac rupture. Int J Cardiol. 2017; 227: 399-403.

[33]M Shahreyar, O Akinseye, M Nayyar, et al. Post-myocardial infarction ventricular septal defect: A comprehensive review, Cardiovascular Revascularization Medicine. 2018; (18):30532-30533.

[34]Kaulfersch C, Daehnert I, Schuler G, Thiele H. Transcatheter closure of postinfarction ventricular septal defects. Minerva Cardioangiol 2007;55(5):693-701.

[35]Goldsweig AM, Wang Y, Forrest JK,et al. Ventricular septal rupture complicating acute myocardial infarction: Incidence, treatment, and outcomes among medicare beneficiaries 1999-2014. Catheter Cardiovasc Interv. 2018;00:11-12.

[36]Mehta LS, Beckie TM, DeVon HA, Simoons ML, Chaitman BR, White HD. Writing Group on behalf of the Joint ESC/ACCF/AHA/WHFTask Force for the Universal Definition of Myocardial Infarction. Acute myocardial infarction in women: A scientific statement from the American heart association. Circulation 2016;133:916-947.

[37]RisseeuwF, Diebels I, Vandendriessche T, et al. Percutaneous occlusion of post-myocardial infarction ventricular septum rupture. Netherlands Heart Journal. 2013; 22(2), 47-51.

\section{Figure Legends}

Figure 1. A PRISMA flow chart depicts the process of search and selection of studies.

Figure 2. Forest plots showing: A) pooled 30- day mortality, B) pooled successful device implantation, C) pooled immediate shunt reduction, D) pooled 30-day mortality of primary closure at acute phase.CI: Confidence Interval.

Figure 3. Forest plots of odds ratio: A) cardiogenic shock, B) NYHA class IV, C) time to PIVSD closure, D) defect size.

Figure 4. The Begg's test: A) 30-day mortality, B) successful device implantation rate.

\section{Table Legends}

Table 1. summarizes the original assessment results of single-arm studies.

More details of basic characteristic in the supplementary table 2 .

Table 2. summarizes the original assessment results of single-arm studies.

\section{Supplementary materials}

Supplementary Figure 1. Forest plots showing: A) pooled 30-day mortality, B) pooled immediate shunt reduction.

Supplementary Table 1. The results of Newcastle-Ottawa scale assessment 


\section{Hosted file}

Figure 1.pdf available at https://authorea.com/users/373239/articles/490992-transcatheterclosure-for-post-infarction-ventricular-septal-defect-a-meta-analysis-of-the-currentevidence

\section{Hosted file}

Figure 2 A-D.pdf available at https://authorea.com/users/373239/articles/490992transcatheter-closure-for-post-infarction-ventricular-septal-defect-a-meta-analysisof-the-current-evidence

\section{Hosted file}

Figure 3 A-D.pdf available at https://authorea.com/users/373239/articles/490992transcatheter-closure-for-post-infarction-ventricular-septal-defect-a-meta-analysisof-the-current-evidence

\section{Hosted file}

Figure 4 A-B.pdf available at https://authorea.com/users/373239/articles/490992transcatheter-closure-for-post-infarction-ventricular-septal-defect-a-meta-analysisof-the-current-evidence

\section{Hosted file}

Table 1.pdf available at https://authorea.com/users/373239/articles/490992-transcatheterclosure-for-post-infarction-ventricular-septal-defect-a-meta-analysis-of-the-currentevidence

\section{Hosted file}

Table 2.pdf available at https://authorea.com/users/373239/articles/490992-transcatheterclosure-for-post-infarction-ventricular-septal-defect-a-meta-analysis-of-the-currentevidence 